\title{
WORKFLOW TOOLS FOR MANAGING BIOLOGICAL AND CHEMICAL DATA
}

THORSTEN MEINL, BERND WISWEDEL, and MICHAEL R. BERTHOLD Universität Konstanz, Konstanz, Germany

\section{INTRODUCTION}

The generation of large amounts of data continues to outpace the ability of automated tools to integrate, transform, and analyze the resulting data repositories. Even though increasingly sophisticated tools are created to handle the enormous piles of heterogeneous information fragments, it is still a big challenge to support a person in extracting the knowledge nugget that in which he or she is actually interested.

In the past, complex analyzes were often performed by highly trained people who wrote and continuously updated and maintained sets of analysis scripts, written in their language of choice, such as Python or R. Such libraries were often not usable for anyone other than the original author, and continued maintenance quickly became impossible without involving the author. From a corporate point of view this was not only highly risky but also made it almost impossible to share knowledge among individuals, let alone different groups. Tools with graphical user interfaces were much more limiting in terms of analytical power but at least also enabled less well-trained users to run standard analyzes on their data. However, every time that a new data set was generated (or an existing one updated) the entire analysis needed to be reexecuted step by step. This process again made it difficult to share expertise among groups and proved to be a huge waste of time, since it required reexecution of standard analyzes over and over again. The best of both worlds is the ability to define an analysis 
process quickly and intuitively and then rerun it automatically on new data while being flexible enongh to make slight modifications, resulting in tools to truly explore and analyze data. In this chapter we discuss some of the most modern, flexible, and powerful tools to support this analytical type of data exploration: namely, tools that allow the user intuitively to model the flow of information through various processing modules. Such workflow or data-pipelining tools have emerged in the past decade or so as the method of choice for quick, flexible integration and analysis of diverse, distributed information repositories while still being able later to reuse the workflow generated to analyze new or updated data. An interesting side effect is the ability to document what has been done and to communicate this visually to other users who may not share the preference for a specific script or analysis language. As a result, workflow tools have emerged as a knowledge-capturing tool as well, allowing companies to convert individuals' experiences into valuable reference knowledge for later use.

Here we focus on four prominent representatives of such tools in the areas of chemo- and bioinformatics. In other areas, such as business intelligence and financial applications, a variety of other workflow or pipelining tools exist; however, they are outside the scope of this chapter. We discuss two commercial representatives (KDE from InforSense ${ }^{16}$ and Pipeline Pilot ${ }^{2}$ from Accelrys) as well as two open-source workflow tools: Taverna $^{22,24}$ and KNIME. ${ }^{4,14}$ These four tools cover the breadth of functionality and address rather different needs: Two are targeted more toward data pipelining, whereas the other two focus primarily on the workflow organization of (distributed) tools. We go into more detail with one of the tools, the open-source Konstanz Information Miner (KNIME), as an example to illustrate and discuss the pros and cons of some of the underlying technical choices. Finally, we demonstrate how such tools can be used based on some examples.

\section{EXISTING WORKFLOW ENVIRONMENTS}

As we mentioned in Section it is highly preferable to model analysis tasks, as they often appear in chemo- and bioinformatics in graphical workflows. Besides the four programs already mentioned, which we discuss in more detail below, there are, of course, other tools, such as Kepler, ${ }^{3,18}$ Insightful Miner, ${ }^{17}$ GridMiner, ${ }^{8}$ DataMining Grid, ${ }^{12}$ LONI Pipeline, ${ }^{19}$ and Triana. ${ }^{34}$ However, they are either not commonly used, do not offer special bio- or chemoinformatics functionality, or require programming skills. Therefore, we concentrate on KNIME, Taverna, Pipeline Pilot, and InforSense KDE.
These programs can be categorized in several ways. First and most obvious is the licensing and/or development model. KNIME and Taverna are open-source projects and may be used completely free of charge, whereas Pipeline Pilot and InforSense KDE are proprietary commercial products. Both approaches have advantages and disadvantages; for new users the uncomplicated and free access to KNIME and Taverna might make them the first choice, whereas for industrial users the supposedly better and longer-term product support of the latter two may be more important.

Another fundamental property is the way in which workflows are modeled and built. A simple linear chain of nodes may already be sufficient for many tasks, but there are many others, where a node requires two different inputs and/or creates more than one output (e.g., a substructure filter which obtains the substructures at the first input and the molecules to be filtered at the second input, subsequently placing the matching molecules at the first output and the nonmatching at the second). Therefore, the theoretical model underlying the workflows is usually a directed acyclic graph, meaning that nodes can have several inputs and outputs, but loops are not allowed. This does not, however, mean that the concept of loops (i.e., iteratively executing parts of a workflow with different data or parameters) is not possible. It is just the data that flow strictly from one end to the other.

Once the workflow has been designed, data are fed into it. How the input data are processed is another conceptual design issue. KNIME, for example, is table based (i.e., the input data are processed completely by any node in the workflow before the results are made available to successor nodes. This may seem to be a disadvantage over the other concept, where single data rows are sent along the pipeline as soon as they become available-Pipeline Pilot uses this concept. However, quite a few processing elements in the workflow still require all the input data anyway (e.g., all kinds of machine learning/data-mining algorithms, such as clustering, decision tree builders, or visualization nodes). Therefore, the implicit parallelism achieved by row-oriented pipelines is only of limited use. In the case of Pipeline Pilot the drawback of this concept is that the processed data are not stored at each node's output, only at special nodes that collect the results. In KNIME the result table of each node is available at all times, even after closing and reopening a workflow (provided that it has been saved, of course). Another disadvantage of pipelining is the limitation to just one input port to nodes; therefore, filtering an input table (or data stream) based on references from another source (e.g., filtering a set of molecules based on a set of substructures) is not done easily. Taverna and KDE operate similar to KNIME in that a node must first 
finish with all data before its successors are executed. Apart from these conceptual differences between the four programs, there are, of course, many other differences, which we discuss further in the following sections.

\section{KNIME}

KNIME is a modular data exploration platform that enables the user visually to create data flows, selectively execute some or all analysis steps, and later investigate the results through interactive views on data and models. KNIME was developed (and continues to be expanded) by the Chair for Bioinformatics and Information Mining at the Universität Konstanz in Germany. KNIME is based on the Eclipse platform, and thanks to its modular API, is easily extensible. Customs nodes and types can be integrated quickly, enabling KNIME to be used not only in production environments but also for teaching and research prototyping. Apart from a large collection of nodes for data preprocessing tasks, it also contains many nodes for data mining and visualization. As already mentioned, KNIME workflows are built from nodes that can be connected in a DAG (directed acyclic graph)-based structure (i.e., a graph without loops). Nevertheless, looping support is available through special loop start and end nodes, which execute the nodes between them (the loop body) a certain number of times or until a specific criterion is fulfilled. This can easily be used for common tasks such as cross-validation, feature selection, or parameter optimization (the loop concept is explained in more detail in Section 3.3). Such subworkflows can be extracted into metanodes, which may then be reused in other workflows.

Another useful concept for interactive data analysis is the hiliting feature, which marks related data rows in all branches of the workflow. We can, for example, consider a node that finds frequent fragments in a database of molecules, such as KNIME's MoSS ${ }^{6,7}$ node. Once an interesting fragment has been found, our interest focuses on the molecules that contain this fragment. By highlighting one (or more) rows in the interactive table, which is connected to the MoSS node's fragment output port, the corresponding rows in the second interactive table, which is connected to any node before MoSS, will also be highlighted. This concept is known as visual brushing in the data-mining world. Figure 1 shows part of this type of workflow, together with the two table views and some hilit fragments or molecules.

Connections in KNIME workflows do not only transport data tables, but may, in fact, transfer arbitrary "port objects" from one node to another. This might include trained predictive models, such as decision trees, support vector machines or regression coefficients, and database connections

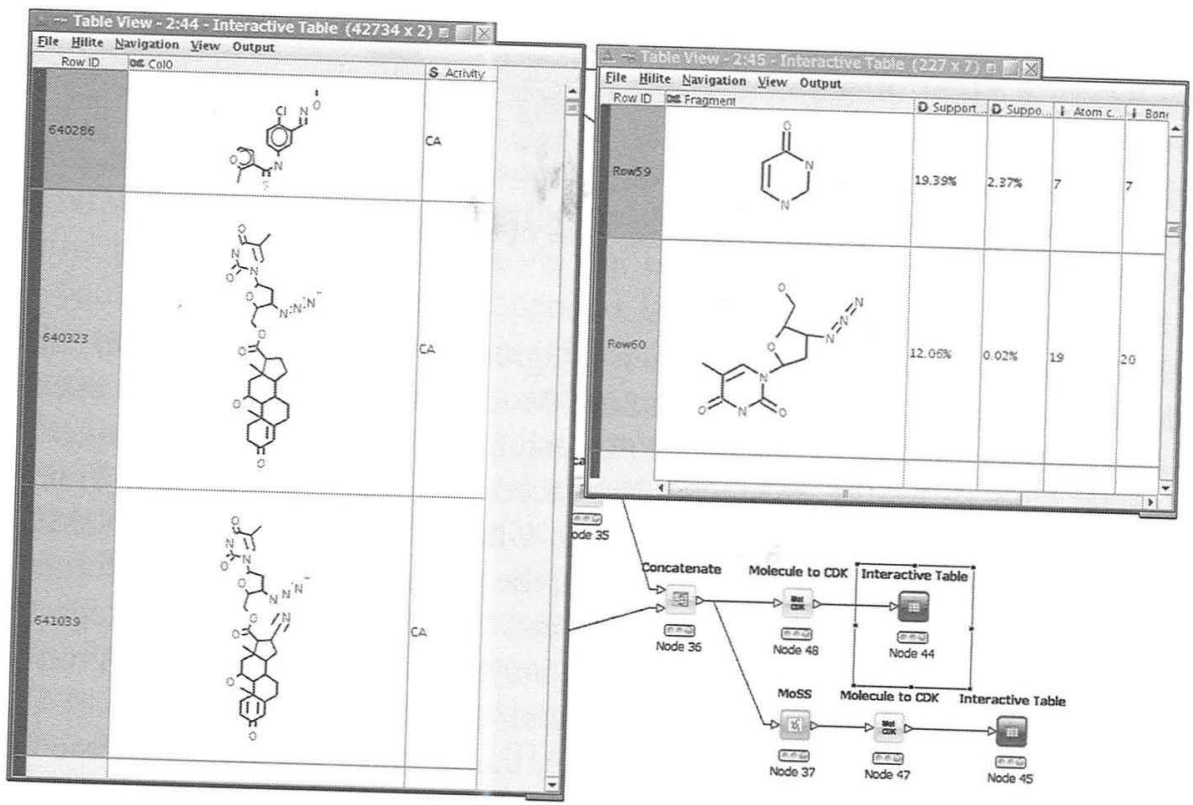

FIGURE 1 Parts of a chemoinformatics-related workflow, demonstrating KNIME's hiliting feature. The MoSS node searches for frequent fragments in a set of molecules. highlight and highlighting several of the fragments found after the MoSS node will also highlight the corresponding rows in the workflow's upper branch.

(again, more technical details are provided in Section 3). The various connection types are indicated by different colors and shapes of the connectors attached to the nodes. KNIME has been designed as a generic data analysis and visual exploration platform and therefore does not offer special bio- or chemoinformatics functionality in its base distribution, which somewhat comprises nodes for data input and output, row- or column-centered manipulation of data tables (filtering, sorting, shuffling, partis etc.) as well as data mining. However, as KNIME is built on the Eclipse ${ }^{13}$ framework, it is simple to add new functionality - usually in the form of new nodes - by providing additional plug-ins. The official KNIME website offers several extensions, the most important (in the scope of this chapter) being several chemistry-related data types (e.g., SDF, Mol2, Deves) and corresponding input/output nodes and the CDK (Chemistry Development $\mathrm{Kit}^{9,30}$ ) plug-in, built on it. By integrating parts of CDK's functionality, molecules can be visualized as two-dimensional (2D) structure diagrams or - if available-as three-dimensional (3D) structures in table views, various properties and descriptors can be calculated and substructure searches can be performed. Figure 2 shows a workflow with 


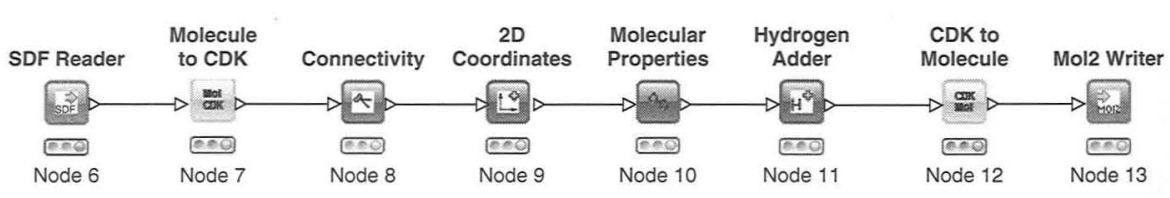

FIGURE 2 workflow showing some of the CDK nodes available.

some available CDK nodes. Other integrated tools are OpenBabel and the already mentioned fragment miner MoSS. In addition to these opensource nodes, several commercial extensions are available. Schrödinger ${ }^{27}$ provides a number of nodes offering access to their chemo- and bioinformatics applications such as LigPrep, Glide, or BLAST from KNIME. Tripos $^{33}$ offers nodes for, among others, the generation of chemical fingerprints, 2D alignments, and conformations, as well as nodes that integrate different DB cartridges. Infocom ${ }^{15}$ has integrated several of ChemAxon's tools, ${ }^{11}$ such as database access, several property calculations, and Rgroup decomposition (for academic use, these nodes are currently free of charge). Symyx ${ }^{31}$ has developed several free nodes for reaction-based enumeration, stereonumeration, or R-group decomposition (however, some of them require a Cheshire license). Molecular interaction fields and chemometrics in pharmaceutical discovery are provided by several nodes from Molecular Discovery. ${ }^{20}$ Treweren Consultants ${ }^{32}$ has packaged its THINK nodes for de-novo derivative generation, structure-based virtual screening (docking), and searching for novel actives using pharmacophores with volume constraints. Several other software providers are working on the integration of their respective tools.

Besides this huge collection of special bio- and chemoinformatics nodes, there are other extensions that may be of use in the workflows, such as basic reporting functionality via the BIRT project, ${ }^{5}$ the integration of the R statistics package, ${ }^{25}$ and Weka ${ }^{35}$ for a vast collection of machine learning algorithms. One of the next releases will also feature several image- and text-processing nodes.

\section{Taverna}

Taverna is another free, open-source software tool that helps users to create scientific workflows, primarily in bioinformatics. Taverna is being developed as part of the UK's myGrid initiative. ${ }^{21}$ Its main focus is on using independent Web services via a common workflow-oriented front end. Nodes (also known as "processors" in Taverna) are discovered dynamically upon startup by querying pre- and user-defined Web service registries. Together with several local nodes, such as input/output, string operations, or dynamic workflow controls, they are shown in a structured node repository. The user can subsequently build a workflow (which is a directed acyclic graph, as in KNIME) by first adding nodes to it and then connecting their various input and output ports. In contrast to KNIME, this cannot yet be performed directly inside the graphical workflow layout but must be done via the list of processors, as the graphical layout is simply a picture generated by GraphViz (this is supposed to change in the next major release). The workflow is represented internally in the Scufl workflow language and can in principle be run separately from Taverna. The use of Web services as main data-processing components has the advantage that the user does not have to install additional software components; instead, they are used out-of-the-box. As a side effect, the workflow can use the remote computing infrastructure, which is usually more powerful than the local computer. Databases and other resources can also be used. This does not come without a cost, however. Changes at the remote side can easily break existing workflows. Also data must be transferred to the remote side first. The result is then transmitted back, which can become a major problem if the data sets are large. Also, Web services may be temporarily unavailable or may simply be removed, which then renders the workflow useless. The data passed along the connections in the workflow do not follow a strict schema like the data tables in KNIME, which are strongly typed (each column has a distinct type). Instead, Taverna uses mime types as "data blocks." More advanced data structures can be built by defining lists or trees of mime types. Unfortunately, this generality makes it impossible to check if the input/output data of two connected nodes matches before executing the workflow. For converting data in such cases, Taverna offers numerous "shim" services, which make sure that two nodes can be used together.

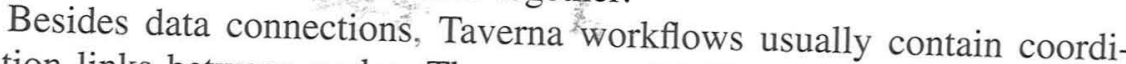
nation links between nodes. These are useful if a node needs the results from another node but where the results are not transferred by data connections but are stored directly on the remote side (e.g., inside a database). A coordination link between these two nodes ensures that the second does not start before the first is finished. Running a workflow in Taverna always entails executing all nodes from beginning to end, whereas other programs often support execution of parts of the overall workflow
as well.

\section{Pipeline Pilot}

SciTegic Pipeline Pilot ${ }^{2}$ is a commercial pipelining tool and-given its wide use-probably the market leader in the chemoinformatics area. 
Components $^{\dagger}$ for data retrieval, filtering, basic analysis, and reporting are available. Although it is known primarily for its chemoinformatics capabilities, it also provides add-ons for, for example, bioinformatics (gene expression profiling, sequence analysis), image analysis (as used, e.g., in high-content screening), chemically aware text mining, and statistics and data modeling. Pipeline Pilot realizes the pipelining paradigm; that is, individual data entities (which correspond to data rows in KNIME, e.g., compounds) are passed on to subsequent components as soon as their processing is finished in one component. There are two principal advantages of this pattern compared to the workflow paradigm, which is implemented by KNIME or Taverna, for example: It implies high scalability by design since no intermediate results need to be cached, and second it allows for an early inspection of the final results. Both benefits come in handy if the entire analysis flow can be streamed (i.e., the components do not iterate the data multiple times, and the focus of analysis is on the final results only). However, this pattern is less well suited for tasks that require multiple iterations on the data as required by many preprocessing or datamining algorithms. It has also shown to be less convenient if subsequent modifications to the pipeline are necessary; for example, the user decides to subbranch from the pipeline or to modify any component in between; this typically requires a rerun of the entire protocol.

Pipeline Pilot uses a client-server architecture in which there are three different client options, which differ in available functionality and commercial support schemes: a professional client for "own" protocol creation and customization as well as protocol sharing, a "lite" client for "own" protocol creation, and a Web port for convenient deployment of existing protocols.

Similar to KNIME, Pipeline Pilot enables a seamless integration of third-party tools. This can be as easy as calling an external executable with dedicated command line flags, the integration of different databases through ODBC protocols, or the call of external Web services. It also allows for a fully fledged integration of libraries using customized components. The latter is commonly used by different independent software vendors, such as ChemAxon, Tripos, and Molecular Networks (the Web page currently lists more than 30 partners). Pipeline Pilot is a commercial software product that addresses many user needs, such as client-server

The term component translates to node in KNIME or processor in Taverna nomenclature, whereas a workflow is known as a protocol in Pipeline Pilot.

‡There are components that allow a buffering of intermediate results in a "finish here, resume later" manner, although these components must be placed in the pipeline beforehand. architecture, role management, Web service integration, and reporting; however, it has drawbacks with respect to usability and is also a relatively expensive solution.

\section{InforSense}

InforSense's workflow editor is part of a larger suite of applications that targets a wide range of applications: from business intelligence to customer relationship management, and from fraud detection to the life sciences. InforSense uses the workflow engine as the central backbone to integrate various different modules, forming the InforSense platform. Some of the resulting applications wrap the workflow so that the user can concentrate on the resulting views: for example, the reporting analytics product.

From the standpoint of this chapter, we are interested primarily in the workflow authoring and execution tool. Similar to most of the other tools discussed here, the editor allows a pipeline of modules that read, manipulate, model, analyze, and finally, write data to be assembled visually. One strength of this platform is the ability to tightly integrate other tools, which is demonstrated by the large list of partners who integrate access to (some of) their tools into this workflow engine, especially in the chemoinformatics domain. However, access to databases and calls to in-database data analysis routines are also supported.

InforSense uses an object-based design paradigm, which can encapsulate data tables as well as more complex objects. This paradigm results in data processing similar to KNIME (i.e., nodes tend to be executed fully before successors become executable). Again, this does enable easy integration of more sophisticated nodes, which rely on seeing the data in its entirety (or repeatedly) in order to, for example, build a model of the data, such as a decision tree or a rule model to predict biological activity.

InforSense's product portfolio encompasses a variety of different extensions, based on this workflow engine. ChemSense, to name one, adds chemoinformatics functionality and allows access to chemical compound databases, specialized data cartridges, and modeling tools from several vendors. Built-in functionality offers basic property calculations and substructure analysis in addition to the visualizations useful for SAR analyzes. Extensions allow text and images and also biological data such as gene and protein expression profiles to be analyzed. However, it is clear that InforSense's expertise lies in the analysis and visualization of complex scientific data sets, while with regard to life science applications, the integration of appropriate partner tools is essential. This also becomes apparent when browsing through the list of industries using this platform. In addition to pharmaceutics and life science, diverse areas such 
as financial services, manufacturing, and communications illustrate the breadth of areas covered.

InforSense offers a commercial product with a wide range of applications. Using partner extensions, a full suite of chemo- and bioinformatics solutions can be addressed; however, to be useful as-is tools, the standalone tools from InforSense lack domain expertise.

\section{TECHNICAL ISSUES}

In the following we describe more technical considerations to be made when designing workflow or pipelining tools, using KNIME as an example. Most of these considerations were inspired by how users expected workflows to behave, whereas others resulted from design decisions that required development in KNIME to be as easy as possible while still enforcing the stability of third-party contributions.

\section{The Node Concept}

As already pointed out, the most important part of each workflow are the data-processing elements, the nodes (Figure 3). Usually, a node has one or several input ports and one or several output ports. Some nodes, such as file readers or writers, have only input/output ports. A node in KNIME consists of up to five components:

- The NodeModel, which processes the data.

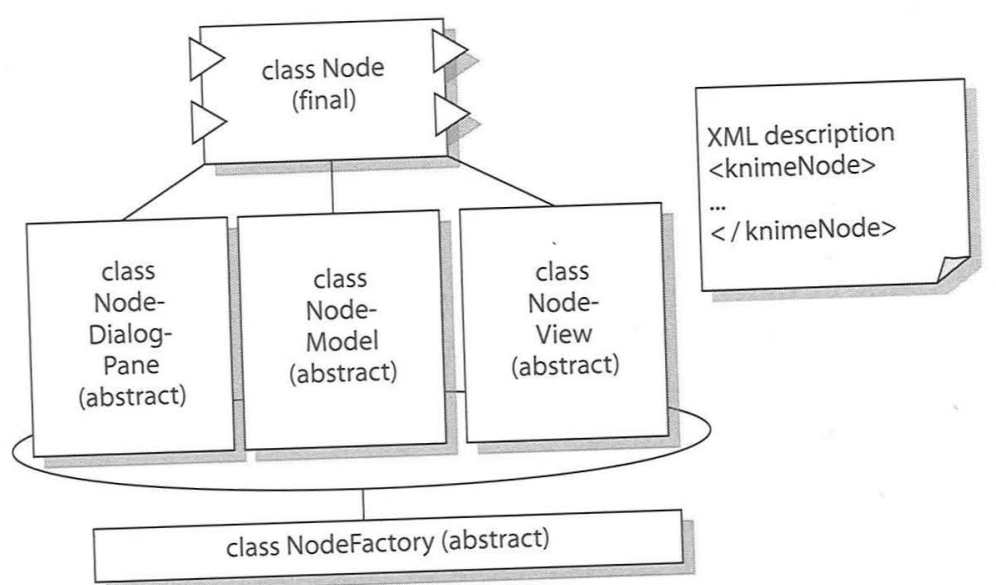

FIGURE 3 A node is made up of the five components shown. NodeView is an optional class.
- The (optional) NodeDialog, which lets the user enter the settings for the node's operation (e.g., which column to use, parameters for the algorithm).

- The NodeFactory, which creates instances of the model, dialog, and view (see below).

- An XML file, which describes the nodes functionality, the parameters the user can adjust, what type of input it expects, and what type of output it creates.

- Optionally, one or more NodeViews, which visualize data or the model built inside the node (e.g., a decision tree or a lift chart).

These are the abstract classes that a node implementor has to extend. Everything else, such as data transfer, invocation of the model, showing the dialog, and so on, is handled by the framework. The most important class, called NodeModel, has two main functions:

- To check if input table structures and user settings are compatible for the specific operation and, if so, to determine the structure of the output table and report it to the successor nodes. This step is performed before any actual data are processed and is called the node's configuration.

- To take the input data, run computation, and create the result table. This step is called its execution.

Separating configuration and execution makes it possible to build a workflow, configure all its nodes, and then afterward, begin (the potentially long-running) processing the data while being sure that output and input data of all connected nodes will match (there are some rare cases in which a node cannot determine the structure of its output table before having seen the actual data). The NodeDialog consists of one or more Swing panels, which can contain other possible Swing elements. Usually, the user adjusts a few settings based on the structure of the input table, which are in turn transferred from the dialog to the model upon configuration or execution. The optional NodeView is also a user-defined Swing component which has access to the NodeModel and can visualize its internal model or the input data (e.g., Scatter Plotter). While executing a workflow the user is usually interested in its current state. Therefore, the status of each node is displayed (waiting for execution, executing, executed, unable to execute). If it is running currently, progress is indicated by a progress bar. Of course, some nodes cannot make such an estimation, but they can at least report how many rows have been processed, or how many solutions (output rows) have already been found. In any case, the user is able to cancel the node's execution at any time. 


\section{Data Handling}

Three major design goals of KNIME have been scalability, ease of use, and a simple extension mechanism. All three principles raise specific challenges to the data-handling mechanism used in KNIME. These challenges and their solutions are discussed in the following sections. To understand them, we first take a brief look at the data structure that is used to pass data through the workflow.

The standard data format is table-based, whereby rows represent different records and columns describe record attributes. As an example, consider a data set of chemical compounds (e.g., represented by SMILES i.e., strings), which are described by numerical measures such as $\log$ $P$, molecular weight, and atom count. The SMILES strings and each of these attributes are represented by individual columns, whereas a row represents a specific compound with its specific attributes. Figure 4 depicts the general architecture and the access methods of this data structure (i.e., the BufferedDataTable). Each table has a predefined table specification (DataTableSpec), which consists of typed and labeled columns (DataColumnSpec). The data, which comply to this specification, are accessible via an iterator (there is no random access on records), which returns records one by one (DataRow elements). The fact that the data are only exposed using an iterator already highlights the fact that tables may have an almost arbitrarily high number of rows (random access in a large collection would have been prohibitively expensive), whereas the column count is typically a small number (ranging from very few to a couple of thousand). KNIME uses a smart caching strategy to swap tables that are too large to fit into main memory. Subsequent iterations on these tables read from the hard disk so that the table is never kept in main memory as a whole (unless a specific implementation of a node decides to do so). KNIME follows a philosophy according to which (temporary) disk space is not a limitation, whereas main memory can be.

Efficient Data Referencing KNIME realizes a workflow pattern that buffers each node's computed outcome. This allows for an inspection of intermediate results (the user can review what has been computed at each individual node even after the entire workflow has finished executing), and second, it offers the possibility of continuing with newly created subbranches after successful execution without the need to rerun the entire workflow. This buffering strategy can be quite expensive in terms of memory or disk usage since many nodes duplicate their input if not handled with caution. KNIME uses table references for nodes that perform columnbased transformations to input tables; that is, it does not copy the input data

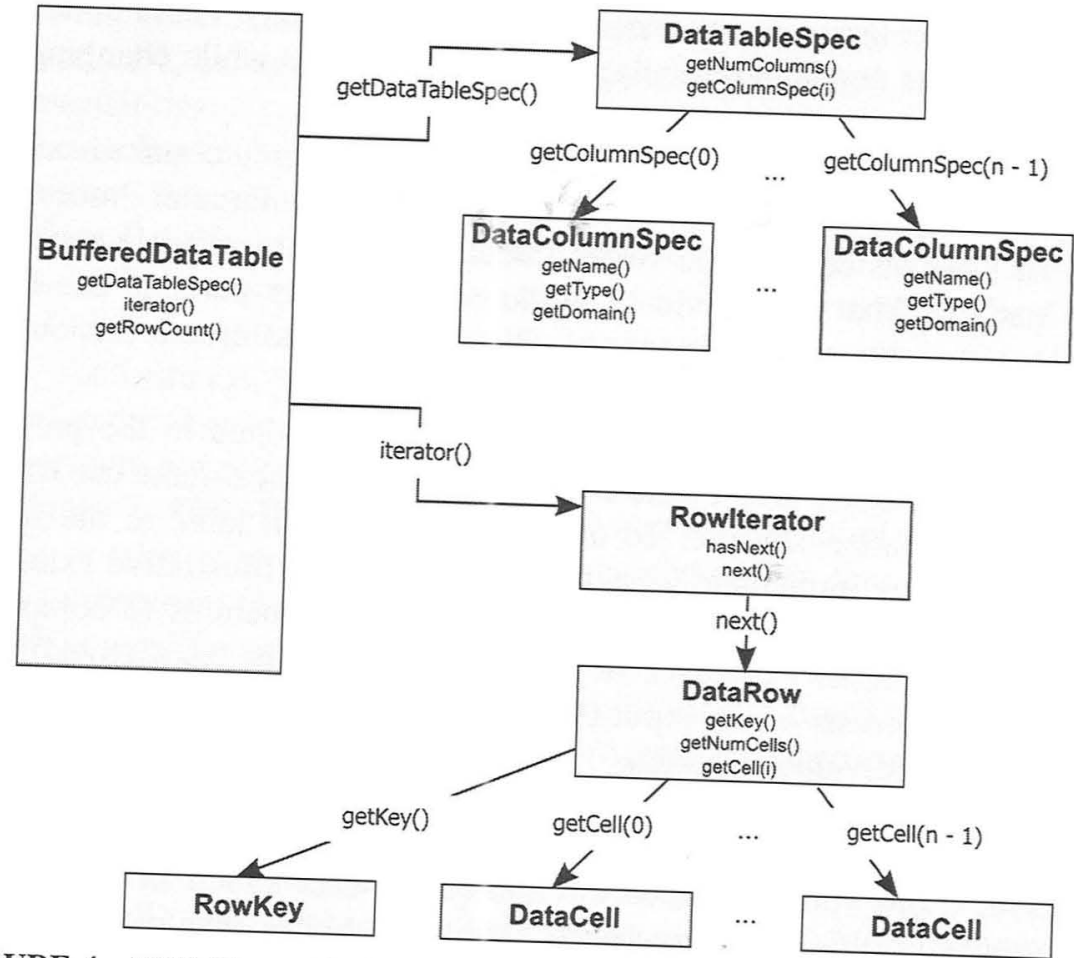

FIGURE 4 KNIME uses BufferedDataTables as the underlying data structure. It has a
fixed number of typed colomns and a fixed number of typed columns and a (possibly very large) number of DataRows. Each
row reprecord in the data set.

but references them and saves only what has changed. In practice, it turns out that a vast majority of nodes that actually perform these types of modifications (i.e., they do not modify the entire anput data but instead append, replace, or modify individual columns). Examples are preprocessing nodes (e.g., column filter), nodes for (chemical) property calculation, and type converters that parse, for instance, numbers or translate chemical represennodes iterating the output another. It is important to note that successor nodes iterating the output of such column transformers will not notice the whereby the unce a BufferedDataTable; instead, they iterate their input, whereby the underlying iterator assembles the "right" data on the fly.

There are other modifiers to which a similar trick can be applied. However, we do not want to delve into too many technical details here but restrict ourselves to brief mention of the following:

1. DataTableSpec replacement. Some nodes perform no data manipulation at all but only change the table specification (e.g., nodes that 
rename columns or set certain properties for them). These nodes do not create any data but only wrap the input table while changing its specification.

2. Concatenation. Nodes that process the input data in chunks in order to exploit today's multicore architecture (see the "Parallel Processing of Data Inside a Single Node") Section make use of table concatenations. That is, individual workers create only parts of the final output table, which are eventually combined to create the output table.

3. Column Append. Specifically, the nodes mentioned in the preceding item often only create new columns and hence make use of the concatenation scheme. To attach the final result table to the input table, they implement an append operation. An illustrative example is a node that generates 3D coordinates for structures (a computationally rather expensive step which can easily be parallelized); the result consists of the input table joined with the concatenated result of the individual workers.

All these operations can be nested arbitrarily, which provides a very powerful framework to reduce the number of data stored in each node. Unfortunately, this trick cannot be applied to nodes that perform rowbased transformation (e.g., sorting or shuffling the data rows), as this changes the order of the rows. For these special cases, the data actually need to be copied. Luckily, there are only a handful of scenarios where this becomes necessary, as most nodes perform calculations or modification based on individual rows.

New Data Types Another design principle of KNIME is its extendability. In Section we discussed the definition of new nodes that perform customized operations. However, this is not the only direction further extension can take. The definition of new data types is often of interest if the focus is on analyzing objects of a very specific nature. Very prominent examples are molecular structures, proteins, and reactions. As a matter of fact, KNIME in its base version has no chemistry support; standard types are limited to a very small set that includes strings, real and integer numbers, bit vectors, and cell collections. Chemistry functionality is available in additional add-ons (called plug-ins), which provide chemistry data types (such as definitions for SMILES, Mol2, SDF, or $\mathrm{PDB}^{\dagger}$ ) and/or nodes that consume or produce data of these nonstandard types. These nodes would be useless without a definition of the respective data type.

$\dagger$ In comparison to Pipeline Pilot, KNIME does not define an "own" chemistry type.
There are more examples of customized data types, which we also discuss in Section 4, including image data, large text documents, and 3D object representations.

The definition of a new data type is simple. It boils down to the definition of common access methods via an interface (extending an interface DataValue), enabling node implementations to retrieve the cell internals, and concrete implementation of a container (called DataCell). The DataValue interface additionally defines common properties of the type: for instance, a comparator, which is used when a table is to be sorted according to a column containing cells of the new type. It can further define a renderer class that is, the functionality to visually depict an object in KNIME views (e.g., to show a 2D depiction of a molecule within a table viewer). DataCell implementation, on the other hand, is used to represent the content and (possibly) to define a custom serializer, which is used for efficient storage and restoration of objects of that type (otherwise, the slower standard Java serialization is used).

Some cell objects, such as proteins, images, or documents, can be relatively large (up to a couple of $\mathrm{MBs}$ ) and need special handling to ensure fast data iterations and efficient disk usage. These types can be represented by blobs (binary large objects); that is, they are not treated like the usual cell objects, which are saved consecutively in a data stream, but instead are saved separately on the hard disk. Further reference to this cell object does not actually store the cell content but, instead, only address information, to ensure that the content is not duplicated. This also has the advantage of enabling faster iterations on tables containing blobs, as they do not get restored as part of the interpretation of the data stream but only on when the cell is actually accessed. The blob concept has shown to be very useful in practice, specifically in the domain of image and text mining (see Section 4).

\section{Loop Support}

We have already mentioned in Section 2 that the workflow structure in all tools discussed here is usually a directed acyclic graph (i.e., there are no loops). Still, some type of iteration concept can be of use in many applications: from common tasks such as cross-validation or feature elimination, to iterating over a list of input files, to even more sophisticated approaches such as using the rows from an input table as parameters for one iteration of the loop (e.g., to find the optimal sets of parameters). Before KNIME 2.0 , this was only possible by using the batch mode, where node parameters are set on the command line before KNIME begins. A "loop" is then simulated by executing the same flow repeatedly with different starting 
parameters. This approach, however, has several drawbacks. First, for each "iteration," KNIME is restarted from scratch, which takes some time. Second, setting the right parameter on the right node via the command line switches is not very intuitive because one the nodes' XML configuration files have to be examined, and third, more complex loops such as feature elimination are not possible at all, because each iteration depends on the results from previous iterations. Therefore, since version 2.0, loops have been supported directly by the workflow manager.

A loop consists of two special nodes, the loop start and end nodes. They are special in the sense that they have access to each other (usually, a NodeModel cannot access any other NodeModel) and that in contrast to the nodes inside the loop, they are not reset while the loop is executing. This is important, because they have to maintain their state during the iterations. For example, the start node of a cross-validation loop has to keep track of the data table partitions that are used as training and test sets, while the end node has to collect the results from each iteration. The decision as to whether the loop should be stopped or another iteration performed is made by the end node. This means that the loop is executed at least once ("do-while" loop). Figure 5 shows the parts of a workflow that contain a feature elimination loop. Conceptually, feature elimination consists of two nested loops (the user sees only one loop in the flow). The "outer" loop is executed as many times as there are features. After each iteration, one of the features (the one that was least important for the trained model) is left out. The "inner" loop determines which of the remaining features is to be removed next by the outer loop. The end node collects the model performance for each "inner" iteration and then decides which attribute is left out for the next "outer" iteration. This process is repeated until only one feature is left. The end node then builds a special feature elimination model, which can be used later by a feature elimination filter, which enables certain columns to be filtered from its input table based on the performance of the model inside the feature elimination loop.

The loop concept becomes even more powerful when it is combined with flow variables. These are simple key-value pairs, which are passed along the connections. Each node may read or write these variables and use them during execution. Inside the dialog the user can then assign a certain variable to a dialog setting. When the node is executed, it takes the value of the variable instead of the value entered in the corresponding dialog component. The last missing piece is a loop start node which takes a data table as input, holds different parameter settings for the node(s) inside the loop, and takes one combination (= row) in each iteration.

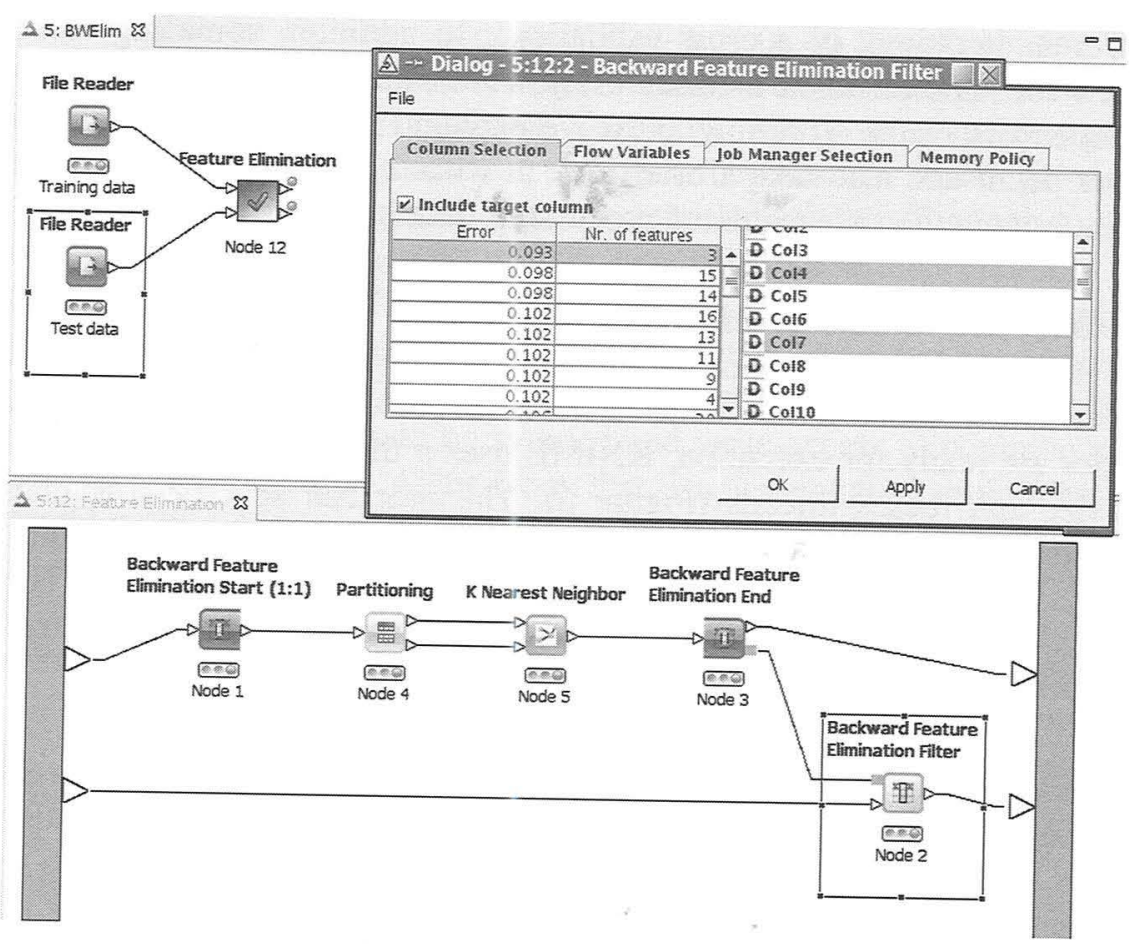

FIGURE 5 Workflow containing a feature elimination loop. After the loop has been fully executed, the feature elimination filter can be used to filter out columns from anothe table, based on the error rates seen while looping.

\section{Parallel Data Processing}

In the field of workflow systems, there are several ways to exploit the parallelism offered by multicore processors or multiprocessor systems with shared memory. The simplest and most obvious is the parallel execution of different branches in the workflow. Each node that is ready for execution can run in its own thread (and is executed on any free processor or core). In the case of real data pipelines (as in Pipeline Pilot), even nodes depending on each other can run simultaneously because as soon as a node has produced one result row it is sent to its successor nodes, which immediately process it further. However, as we explained in Section 2, this inherent parallelism cannot be used throughout the entire workflow. Also, if the runtime per row is very different for the nodes involved, the achievable speedup is quite limited.

In KNIME, the next, more advanced approach is to process the data rows of the input table in parallel. Finally, the most sophisticated method of parallelization is to execute entire subworkflows in parallel (e.g., the 
To achieve an equally distributed load among all threads on the one hand and a low overhead on the other, a suitable balance between the size and number of chunks is important. Currently, four times as many chunks are created as available threads in the pool. The abstract model provided by the framework calls a method in the concrete subclass for each row of the input table, which then returns the new cells that are appended to the row. In the final phase the results are merged (cf. the Section "Data Handling") and a complete output table is built. The programmer need only be aware that the code is called by several threads concurrently. Therefore, synchronized blocks should be avoided and write-access to common data must be used carefully. Apart from that, implementation resembles the normal node's API. Almost the same usage model applies in cases where the number of output rows differs from the number of input rows or when the structure of the output table is completely different from the input table structure. Again, the framework invokes a special implementation for each row of the input data. This time, however, a (possibly empty) set of complete rows must be returned. Again, the framework takes care of merging the final results. The threaded nodes have a slight overhead that comes from splitting the input tables and merging the results. This depends largely on the size of a row (the number of columns and the size of the objects in the cells) and on the input/output speed. Generally, however, this overhead does not significantly impair performance.

Besides this local thread-based parallelization, we are currently working on distributing the different chunks as jobs into a local cluster. The user can select how many subjobs should be created (or the number of rows per job), and the framework keeps track of splitting the input table, creating the cluster jobs, and finally, merging the results. Behind the scenes a small workflow is created that contains just the node(s) that should run on the cluster. On the cluster, KNIME is started in batch mode and runs the workflow with one of the input table chunks. Ideally, this distributed execution should offer the same "feeling" as locally executed nodes (i.e., it should be possible to cancel them and proper progress should be reported to the user). Also, users should be able to close their KNIME session and reopen it later and still obtain the results from the cluster jobs (if they have already finished) or monitor the current progress.

Parallel Processing of Subworkflows Earlier we explained the concept of loops that repeatedly execute parts of a workflow. A popular use case is cross-validation to estimate the stability of a trained model. Figure 7 shows an example of the use of a cross-validation node. First, the data are read and then fed into the cross-validation loop. Inside the loop the $\mathrm{X}$-partitioner is responsible for splitting the data into training

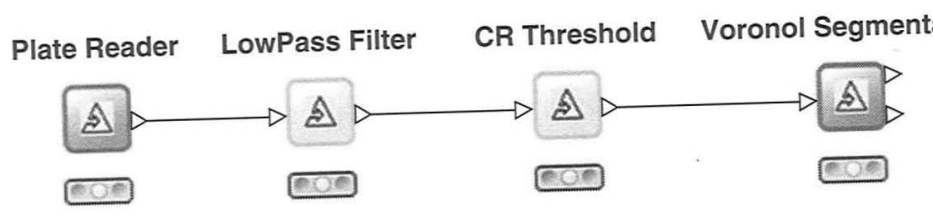

Cell Images

Two small workflows with threaded nodes. 


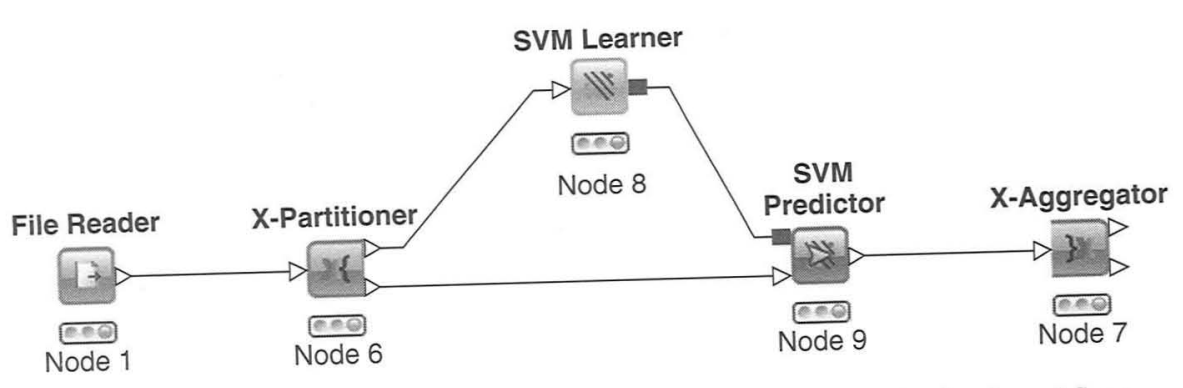

FIGURE 7 Workflow with a cross-validation node and its internal subworkflow.

and tests sets, whereas the X-aggregator node collects the results and creates statistics for each iteration. Inside the loop, the user has to insert at least two nodes that build a model based on the training data and classify the test data using the model. In the example workflow shown, the SVM learner node is used, which trains a support vector machine.

Using support vector machines is relatively time consuming, even for small data sets, and becomes increasingly so if there are 10 or more iterations in a cross-validation node. In such a case, all iterations are independent of each other, and partitioning into training and test data must be identical (i.e., in each iteration another 9 of 10 partitions are used for training, and the remaining partition is used for testing). Therefore, it is quite natural to parallelize the execution of single iterations. Unfortunately, this time, more effort is needed from both sides, the KNIME framework and the node programmer. The fact that a single node is now run in several threads at the same time, especially, poses quite a lot of problems. That these difficulties are solvable has already been demonstrated in a prototype implementation. ${ }^{29}$ The fact that this requires some major changes to the internal execution strategy of workflows and to the node implementations themselves has kept us from integrating it into the official KNIME releases so far.

\section{Integration of Third-Party Contributions}

We mentioned in Section 2 that besides the CDK integration, the base KNIME distribution does not offer much bio- or cheminformatics-related functionality. Most of the nodes available come from third-party vendors. Because KNIME is based on Eclipse, it is very easy to add additional nodes (or other functionality) by using Eclipse's plug-in mechanism. The KNIME core offers extension points to which other plug-ins can be attached. The plug-in offering the extension point subsequently reads out all attached extensions; in the case of KNIME, these are usually additional nodes. The nodes are then added into the node repository automatically and are usable like any other KNIME node. This extension mechanism allows for a lot more than simply being able to add nodes. In the current development version a plug-in can even change the way that single nodes are executed by providing a special node executor. The distributed processing of a node inside a cluster that we described earlier is realized by an additional plug-in.

Quite often, users and vendors want to integrate existing programs or scripts into KNIME. For very simple scripts, KNIME offers the external tool node, which creates a CSV file from the input table, launches an external program with this file as an argument, and finally, reads a result file back in. For small amounts of table-structured data, this works well, but it does not work for large numbers of molecules in SDF format, for example (which has a special internal structure). In such cases, dedicated nodes have to be programmed to create the program's input files in the right way and to parse the results in the correct manner back into KNIME. However, this file-based transfer of data is far from optimal. Ideally, the external program could work inside a "pipe" created by the KNIME node. It is given input data on its standard input and writes the results to standard output, whereupon KNIME reads them back in. This not only renders the intermediate files obsolete but provides for much better progress reporting, as KNIME simply counts the rows it has already piped into the external program (or the number of results it has received). However, usually the external program has to be adapted for this type of piped processing. Unfortunately, currently, most vendors refrain from modifying their existing software. However, with regard to open-source software, this should be a manageable task.

\section{EXAMPLE FLOWS AND USE CASES}

In this section we show some example workflows that come from three different research areas. The first, a chemoinformatics-related use case is the realization of a classical virtual high-throughput screening proto$\mathrm{col}$; the second is a bioinformatics application, the automated analysis of cell assay images; and the third deals with the more general issue of text mining in scientific publications. The workflows presented are all implemented with KNIME, but in principle, similar workflows should be possible in the other workflow environments as well.

\section{Virtual High-Throughput Screening}

In traditional high-throughput screening (HTS), hundreds of thousands of compounds are automatically tested for their reaction in combination with 
a given target protein. Usually, the degree to which a compound inhibits the activity of the protein is measured. Molecules with high inhibition rates are then tested further in smaller assays, where the exact concentration at which they prevent $50 \%$ of the protein's activity (the $\mathrm{IC}_{50}$ value) is determined.

HTS is quite an expensive and time-consuming process which severely restricts the number of compounds that can be tested. Therefore, quite a few computational methods have been developed that try to predict a molecule's activity without performing real tests. Common approaches are:

- Protein-ligand docking, where a compounds is "docked" into the protein's active site and the total energy of the bound ligand-protein complex is taken as a measurement for the assumed activity (the lower the energy the better it should bind to the protein).

- Several ligand-similarity methods, such as Feature Trees, ${ }^{26}$ Cofea, ${ }^{1}$ or Shape Signatures, ${ }^{36}$ that compute a similarity measure based on the molecules' properties, driven by the assumption that similar molecules show similar reactions.

- Pharmacophore searches, where abstract properties (charge, volume, proton donors/acceptors) of known active (or inactive) compounds describe a $3 \mathrm{D}$ search pattern with with which the entire database is scanned.

- Simple target-independent molecular descriptors, which can also be used to compute the similarity between molecules.

These approaches can now either be used to replace HTS (which is usually not applied) or as a preselection step in which a set of compounds from a virtual library is selected that is processed afterward by traditional HTS. Figure 8 shows a workflow for the second approach.

On the left, data are read into the workflow. This not only includes the molecular structures, which are later processed by some Schrödinger nodes, but also text files containing output from a Feature Trees comparison and from Cofea. This can be slightly more complex, as in the case of the feature tree similarities. In the lower part of the workflow, some of the Schrödinger nodes essentially perform a docking of the virtual library into a prepared protein. Then the scores and similarities from the various sources are joined together so that each row corresponds to one molecule and its attributes are in the columns. The following meta node computes a number of very simple combined scores by taking the maximum (or minimum) scores of a certain approach (e.g., the maximum of all Cofea

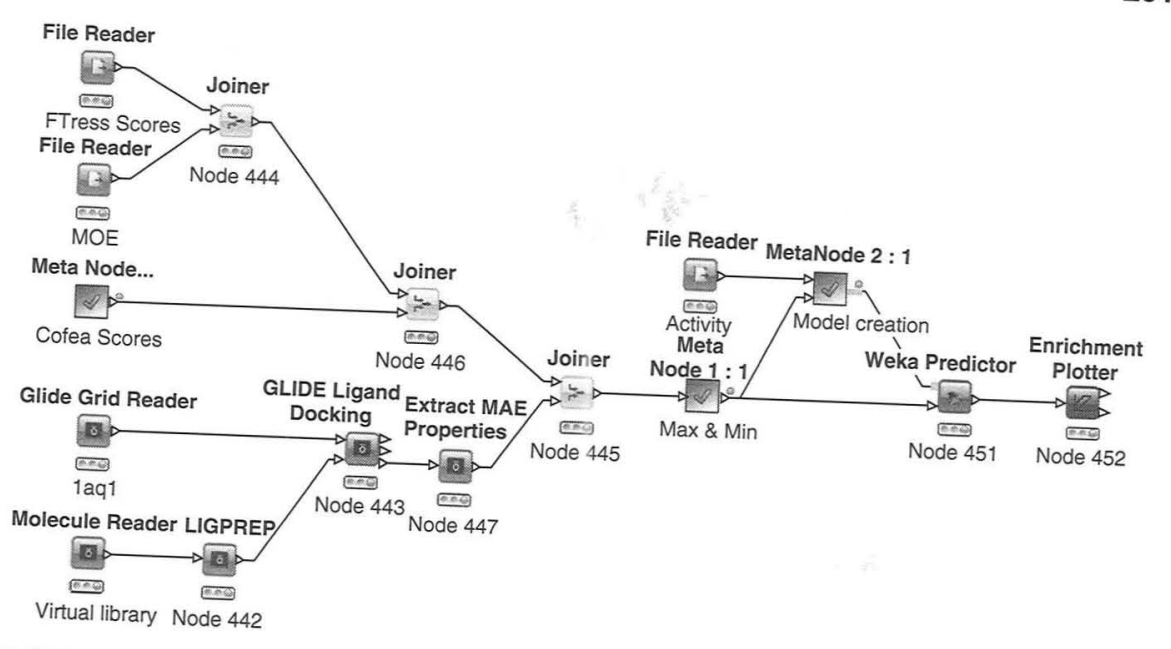

FIGURE 8 Workflow used for preselection compounds from a huge virtual library prior to HTS.

scores from comparisons to various references). Then another meta node follows, which contains a cross-validation loop (see Figure 9). This part of the workflow is used to measure the quality of the built model if activities for some compounds are already known. A support vector regression algorithm is used to predict the $\mathrm{IC}_{50}$ values, which later can be used to sort the molecules. The cross-validation loop splits the input data into sort ing and test sets, uses the training set to build the model, and predicts the value for the test set. The loop's end node collects the results from

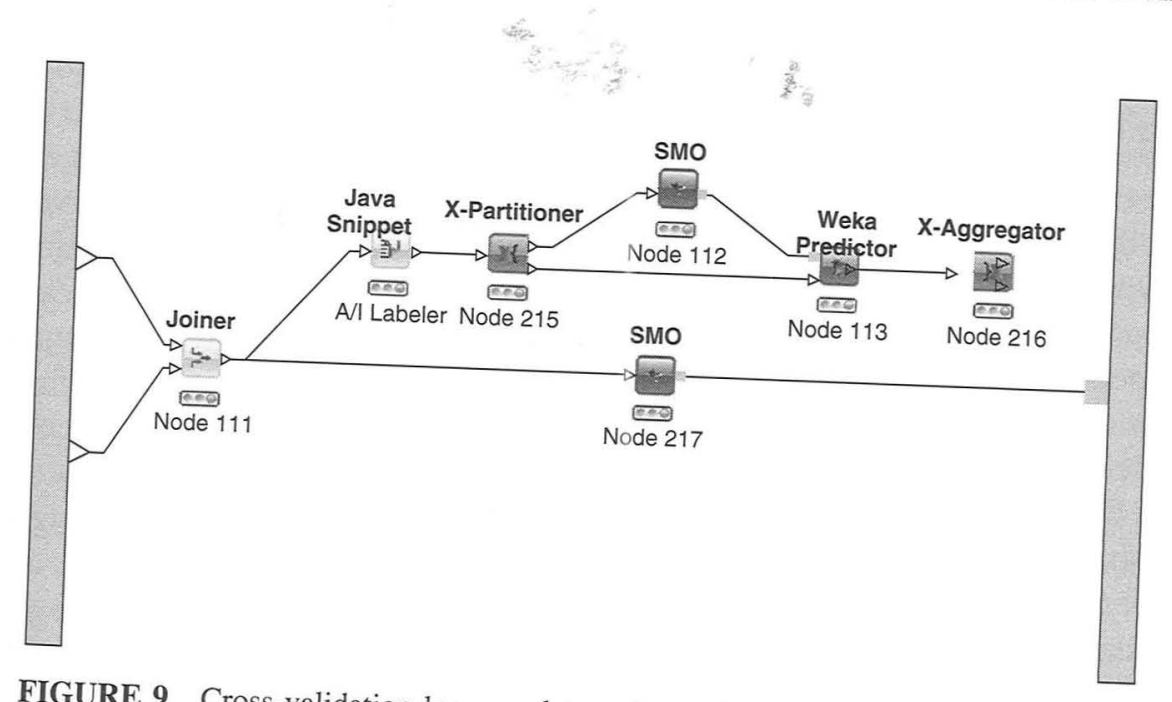

FIGURE 9 Cross-validation loop used to estimate the quality of the trained model. 


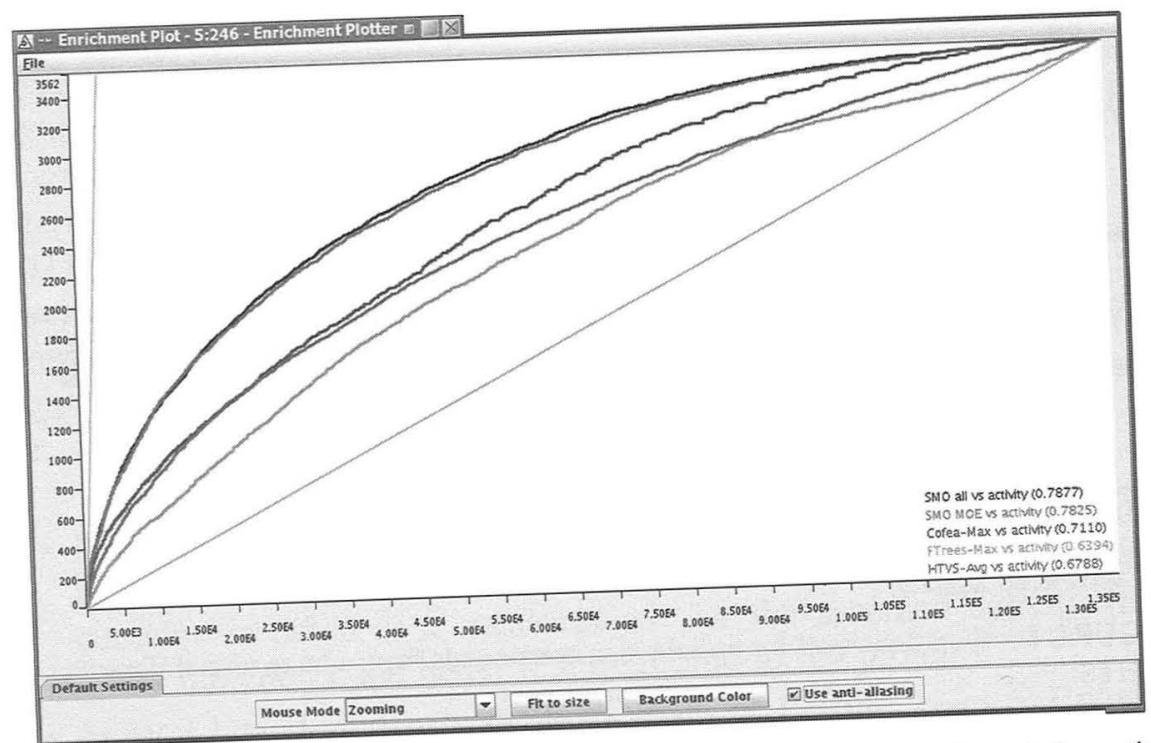

The enrichment plotter shows all molecules on the $x$-axis and the active FIGURE 10 The enrichment plotter show first sorting all molecules molecules on the $y$-axis. The different graphs are created by fiscovered among the first based on the prediction and then plotting the number of actives discovered among thereas $x$ ranked molecules. The light-gray line on th

the diagonal indicates random enrichment.

each iteration and finally creates a statistic (error rate) for each iteration. The prediction results can subsequently be visualized graphically via the enrichment plotter (see Figure 10) as well.

\section{Cell Assay Image Analysis}

The second example workflow we present is used to analyze cell images. In the last few years the development of high-throughput imaging instruments (e.g., fluorescence microscope cameras) resulted in them becoming a promising tool to study the effect of drug candidates on different cell types. These devices are able to produce hundreds of thousands of images per day. One goal of the cell assay image analysis is to label a few selected cell images by hand and to automatically label the vast majority of the images afterward. To obtain a classification of one image, it is segmented into small subimages, each containing one cell of the original image. Segmentation allows the cells to be considered separately in order to distinguish between different reactions of cells in the same image. When most of the small subimages are classified, a classification of the original image can be made by a majority decision.

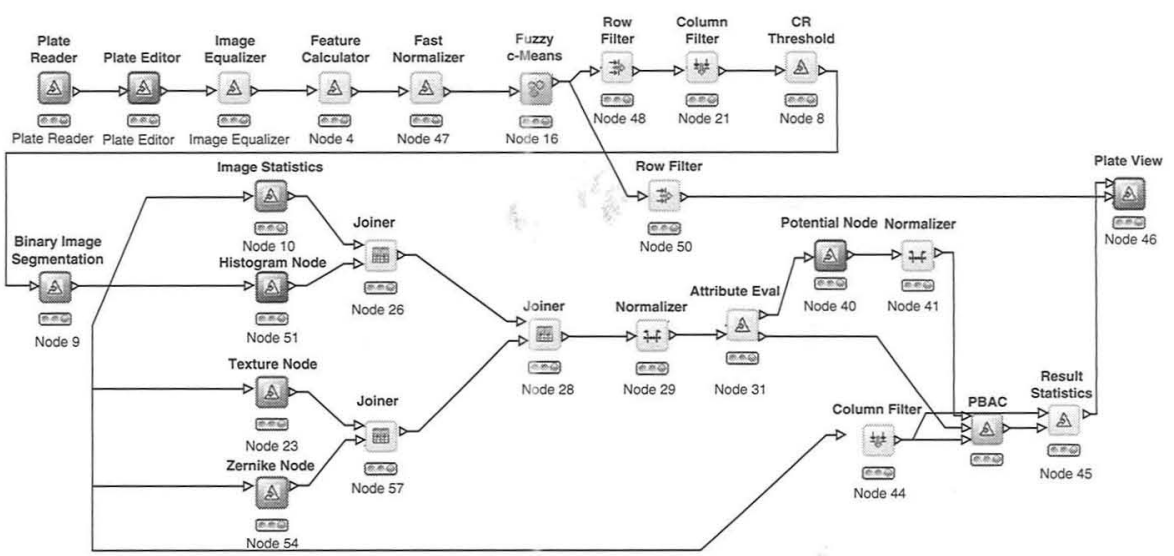

FIGURE 11 Workflow demonstrating the analysis of cell assay images by various image-processing nodes. (See insert for color representation of the figure.)

The workflow shown in Figure 11 consists of three major parts: the segmentation part, the feature extraction part, and the classification part. First, the raw images from the used plates are read in via the plate reader and the plate editor. Then the images are equalized, which means that their gray values are scaled to the complete range available. After that, several features such as histograms or textures are computed for each image as a whole. After normalizing the images' histograms, they are roughly clustered; some clusters are removed afterward (e.g., if they are too noisy). Then the images are segmented into small subimages, each containing a single cell. Obviously, the resulting number of data points is very large, because the thousands of images are segmented into small subimages. This results in an order of millions of images. The next nodes in the flow compute such features as texture, Zernike shapes, and peaks for each of the small single-cell images, which later are joined together in one big table. The last part of the workflow consists of the classification of the images, which in this case is performed using an active learning approach. ${ }^{10}$ Briefly, this means that the user has to classify some (carefully selected) images first and then the algorithm only asks the user for images about which it is uncertain. The final classification of the complete images is obtained by classifying each individual cell within the given image. Each cell is assigned to a cluster and its corresponding class. The proportion of the distribution of the various classes is the decisive factor for classifying the entire image. If a clear majority decision can be made, the image does not need to be considered further. Figure 12 shows the plate view, in which the wells are assigned different classes indicated by the colors. 


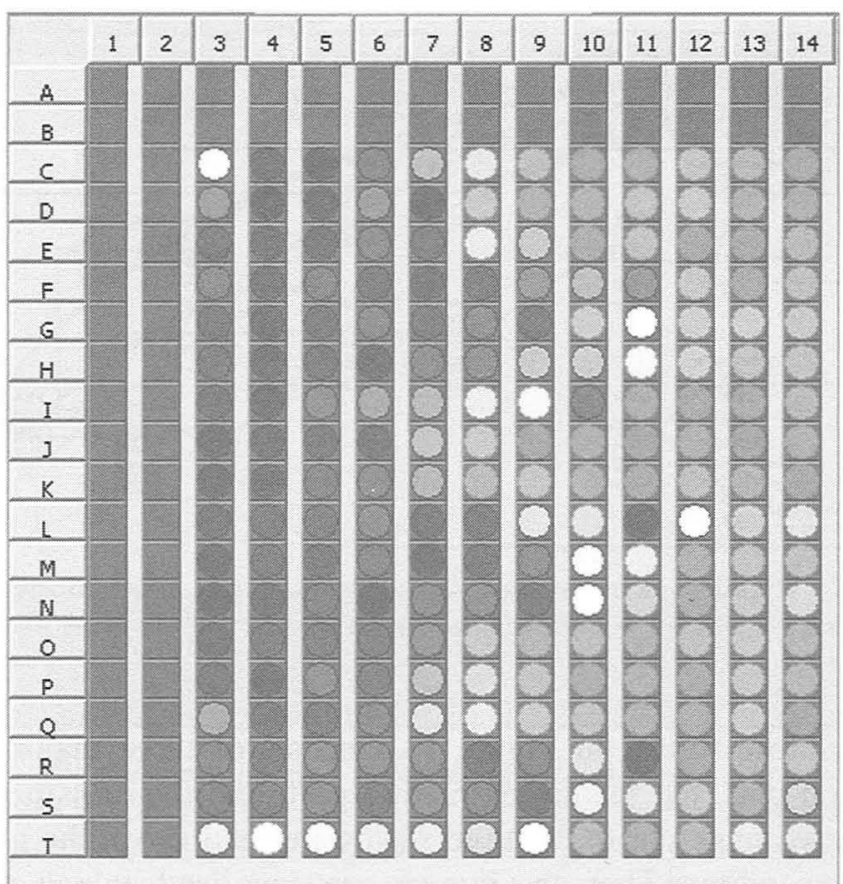

FIGURE 12 The plate viewer provides a graphical view of the classification results. Each point is a separate well on the plate, with the color indicating the class of the cell culture contained.

\section{Text Mining in Scientific Publications}

Text mining is a research area where unstructured texts are analyzed automatically and transformed into data structures that can be used with classical data-mining techniques such as clustering or even classification. Problems arising in text mining and natural language processing (NLP) are manifold: for example, part-of-speech tagging, stemming, named entity recognition (i.e., assigning words to different categories, such as gene or protein), and concept or keyword extraction. There is an extension for KNIME, which enables analysis of text documents, that contains special data types, such as documents, terms, sentences, and tags, together with a bunch of nodes. Figure 13 shows a big flow that processes PubMed abstracts for later clustering and classification. The two nodes at the top left take a simple query string and fetch the matching abstracts from PubMed. In the example two different document categories are retrieved, one dealing with "Human" and "Aids," the other with "Mouse" and "Cancer." After concatenating the two tables, which contain each matching document as a document cell in a row, first biomedical entities such as

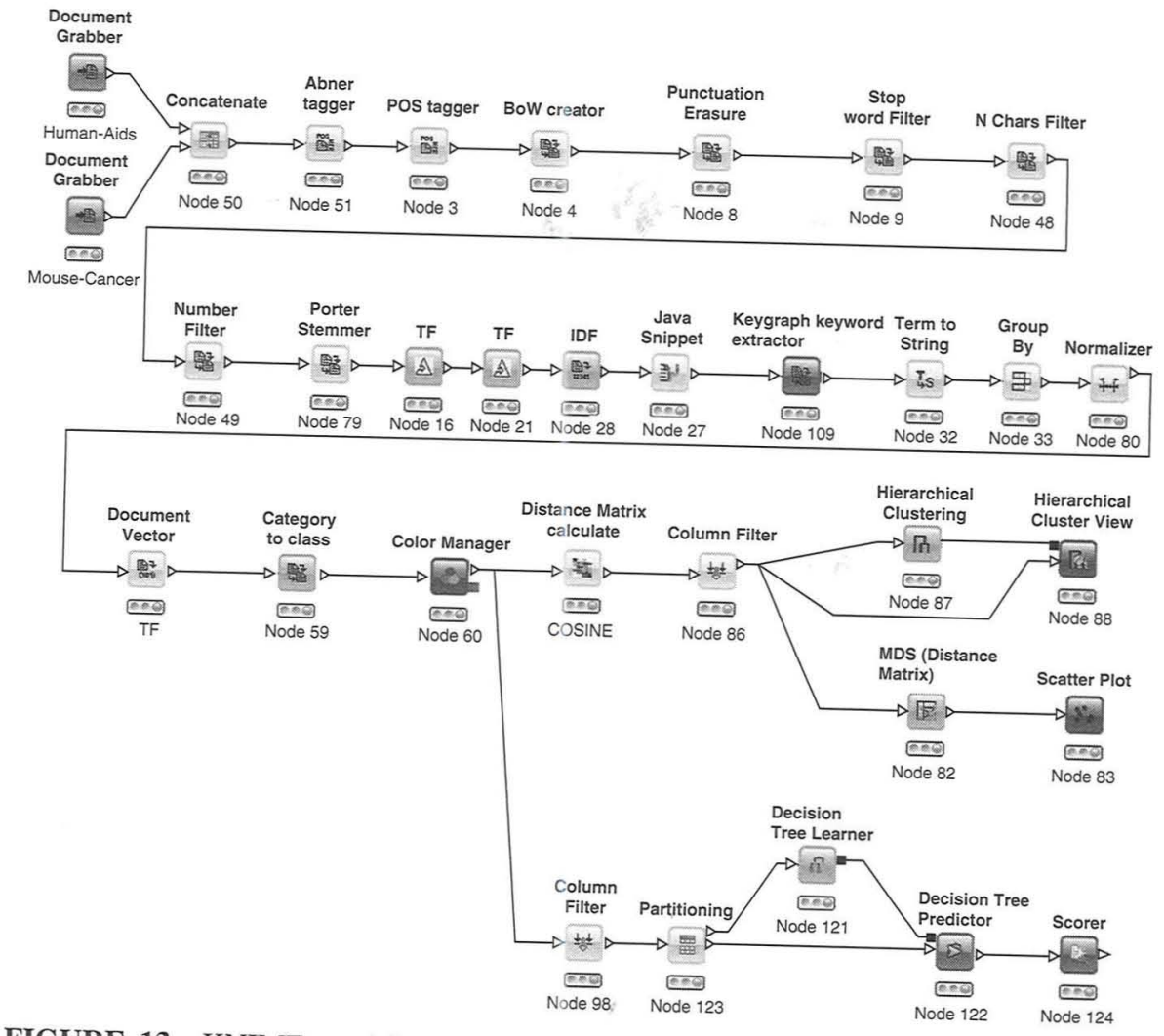

FIGURE 13 KNIME workflow showing the use of the text-mining extension. After several preprocessing steps, the documents are clustered and a decision tree for late classification of new documents is built.

"gene," "protein," or "cell line" are recognized using ABNER, ${ }^{28}$ and then parts-of-speech (verb, noun, etc.) are assigned. Next a "bag of words" is created (i.e., tuples of single words and documents). The next four nodes remove terms that are generally useless for further analysis, such as stop words ("and," "is," "the," ...), punctuation characters, words that are too short, or numbers. The Porter stemmer then stems all terms and outputs only their word roots, which usually reduces the number of terms. Then relative and absolute frequencies for each term in each document are computed together with the inverse document frequency. The Java snippet node computes the $T F * I D F$ value, which is the relative term frequency multiplied by its inverse document frequency. Next, the important keywords are extracted from the documents, in this case using a graphbased approach. ${ }^{23}$ The following nodes change the table format so that it contains a document vector for each document, where the entries in the 
Boolean vector represent the extracted keywords and indicate whether or not a word appears in the document. The category to class node adds to each row (either "Human-Aids" or "Mouse-Cancer") a column containing the class of the document, and the color manager adds color information based on the class assigned to each row (the colors can be chosen in the dialog). The last part of the workflow is to demonstrate two possible ways to build models from these document vectors. In the two upper branches, unsupervised models are built-a hierarchical cluster model and a 2D projection with MDS - using a precomputed distance matrix. For highdimensional bit vectors such as the document vectors, it is preferable, for example, to use the cosine distance instead of the standard Euclidean distance. The two models can be visualized later. By using the appended color information, it is easy to determine the quality of the model just by looking at it. In the cluster dendrogram, branches should consist mainly of items from the same class at the leaves, and in the MDS, plot points with equal colors and shape (= class) should group together. This works quite well for the example data used, as can be seen in Figure 14 (we do not show the cluster dendrogram, as the different classes are only visualized

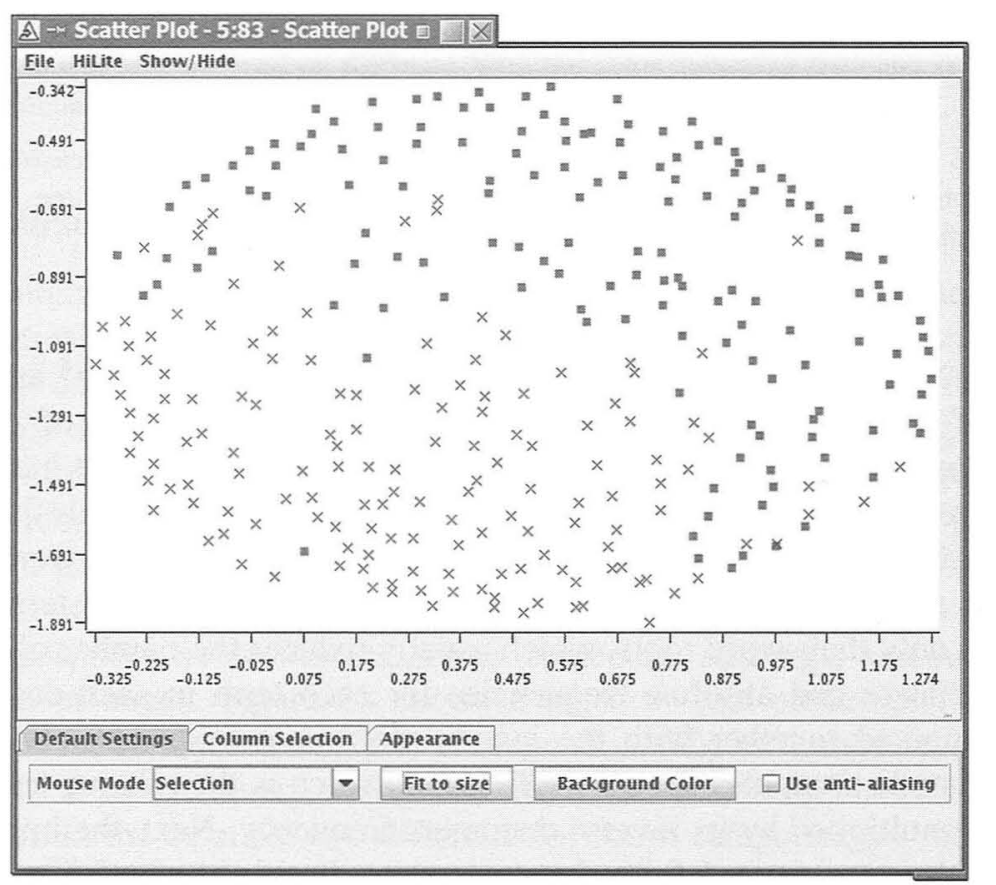

FIGURE 14 Two-dimensional projection of the document vector space from the MDS algorithm. The two document classes (squares and crosses) separate quite well.

\begin{tabular}{|c|c|c|}
\hline D $\rightarrow$ Confusion Matrix - 5:124 & - Scorer [ & $x$ \\
\hline File Hilite & & \\
\hline $\begin{array}{l}\text { Document class \Prediction (DecTree) } \\
\text { Human-Aids }\end{array}$ & $\begin{array}{l}\text { Human-Aids } \\
74\end{array}$ & $\frac{\text { Mouse-Cancer }}{1}$ \\
\hline Mouse-Cancer & 12 & 63 \\
\hline Correct classified: 137 & Wrong clas & sified: 13 \\
\hline Accuracy: $91.333 \%$ & Error: 8 & $.667 \%$ \\
\hline
\end{tabular}

FIGURE 15 The scorer shows the confusion matrix of the prediction and the classification error and accuracy.

by small color points and cannot be distinguished well if printed in this format).

The lower branch performs supervised training of a decision tree by using the available category information. The built tree can be used later to classify unknown documents. In the example this is simulated by splitting the entire document table into two parts, one for training the other for testing. The scorer node at the end shows a confusion matrix and the error rate of the prediction (see Figure 15).

\section{Acknowledgments}

We want to thank the members in our group who have provided valuable comments while preparing this chapter (besides having implemented various parts of KNIME), especially Kilian Thiel for providing the text-mining flow and Nicolas Cebron for the cell assay image analysis example. Special thanks goes to Nycomed for the vHTS and cell image data used in the example flows. Last but not least, Heather Fyson did a great job of improving readability and spelling.

\section{REFERENCES}

1. Badreddin Abolmaali, S. F.; Wegner, J. K.; Zell, A. The compressed feature matrix: a fast method for feature based substructure search. J. Mol. Model. 2003, 9(4), 471-490.

2. Accelrys. Pipeline Pilot. http://accelrys.com/products/scitegic/.

3. Altintas, I.; Berkley, C.; Jaeger, E.; Jones, M.; Ludäscher, M.; Mock, S. Kepler: an extensible system for design and execution of scientific workflows. In Proceedings of the 16th International Conference on Scientific and Statistical Database Management, 2004, pp. 423-424. 
4. Berthold, M. R.; Cebron, N.; Dill, F.; Di Fatta, G.; Gabriel, T. R.; Georg, F.; Meinl, T.; Ohl, P.; Sieb, C.; Wiswedel, B. KNIME: the Konstanz information miner. In Proceedings of the 4th Annual Industrial Simulation Conference, Workshop on Multi-agent Systems and Simulation, 2006.

5. BIRT. Business Intelligence and Reporting Tools. http://www.eclipse. org/birt/.

6. Borgelt, C.; On canonical forms for frequent graph mining. In Workshop on Mining Graphs, Trees, and Sequences at PKKD 2005, pp. 1-12.

7. Borgelt, C.; Berthold, M. R. Mining molecular fragments: finding relevant substructures of molecules. In Proceedings of the IEEE International Conference on Data Mining. IEEE Press, Piscataway, NJ, 2002, pp. 51-58.

8. Brezany, P.; Janciak, I.; Min Tjoa, A. GridMiner: an advanced support for e-science analytics. In Data Mining Techniques in Grid Computing Environments. Wiley, Chichester, UK, 2008, pp. 37-56.

9. Chemistry Development Kit. http://cdk.sf.net/.

10. Cebron, N.; Berthold, M. R. Adaptive active classication of cell assay images. In Knowledge Discovery in Databases: PKDD 2006 (PKDD/ECML), Vol. 4213. Springer-Verlag, Berlin, 2006, pp. 79-90.

11. ChemAxon. http://www.chemaxon.com/.

12. DataMining Grid. http://www.datamininggrid.org/.

13. The Eclipse Project. http://www.eclipse.org/.

14. Nycomed Chair for Bioinformatics and Information Mining at the University of Konstanz. KNIME: Konstanz Information Miner. http://www.knime.org/.

15. Infocom Corporation. http://www.infocom.co.jp/index_e.html.

16. InforSense. InforSense KDE. http://www.inforsense.com/kde.html.

17. Insightful. Insightful Miner. http://www.insightful.com/products/iminer/ default.asp.

18. Kepler Project. http://www.kepler-project.org/.

19. UC Los Angeles Laboratory of Neuro Imaging. LONI Pipeline. http:// pipeline.loni.ucla.edu/.

20. Molecular Discovery Ltd. http://www.moldiscovery.com/.

21. myGrid. http://www.mygrid.org.uk/.

22. myGrid Project. Taverna Project Website. http://taverna.sourceforge.net/.

23. Ohsawa, Y.; Benson, N. E.; Yachida, M. KeyGraph: automatic indexing by co-occurrence graph based on building construction metaphor. In $A D L$ '98: Proceedings of the Advances in Digital Libraries Conference. IEEE Computer Society, Washington, DC, 1998, pp. 12-18.

24. Oinn, T.; Greenwood, M.; Addis, M.; Alpdemir, N. M.; Ferris, J.; Glover, K.; Goble, C.; Goderis, A.; Hull, D.; Marvin, D.; et al. Taverna: lessons in creating a workflow environment for the life sciences. Concurrency Comput. Pract. Exper. 2006, 18(10), 1067-1100.

25. R Project. The R Project for Statistical Computing. http://www.r-project.org/.
26. Rarey, M.; Dixon, J. S. Feature trees: a new molecular similarity measure based on tree matching. J. Comput.-Aided Mol. Des. 1998, 12(5), 471-490.

27. Schrödinger, Inc. http://www.schrodinger.com/.

28. Settles, B. ABNER: an open source tool for automatically tagging genes, proteins, and other entity names in text. Bioinformatics 2005, 21(14), 3191-3192.

29. Sieb, C.; Meinl, T.; Berthold, M. R. Parallel and distributed data pipelining with KNIME. Mediterr. J. Comput. Networks 2007, 3(2), 43-51.

30. Steinbeck, C.; Hoppe, C.; Kuhn, S.; Floris, M.; Guha, R.; Willighagen, E. L. Recent developments of the chemistry development kit (CDK): an opensource Java library for chemo- and bioinformatics. Curr. Pharm. Des. 2006, 12(17), 2111-2120.

31. Symyx. http://www.symyx.com/.

32. Treweren Consultants. http://www.treweren.com/.

33. Tripos, L. P. http://www.tripos.com/.

34. Cardiff University. Triana. http://www.trianacode.org/.

35. Witten, I. H.; Frank, E. Data Mining: Practical Machine Learning Tools and Techniques, 2nd ed. Morgan Kaufmann, San Francisco, 2005.

36. Zauhar, R. J.; Moyna, G.; Tian, L. F.; Li, Z. J.; Welsh, W. J. Shape Signatures: a new approach to computer-aided ligand- and receptor-based drug design. J. Med. Chem. 2003, 46(26), 5674-5690. 